\title{
Displacement of fovea toward optic disk after macular hole surgery with internal limiting membrane peeling
}

This article was published in the following Dove Press journal:

International Medical Case Reports Journal

16 October 2017

Number of times this article has been viewed

\section{Kouichi Ohta \\ Atsuko Sato \\ Nami Senda \\ Emi Fukui}

Department of Ophthalmology, Matsumoto Dental University, Shiojiri, Nagano, Japan
Correspondence: Kouichi Ohta Department of Ophthalmology, Matsumoto Dental University, 1780 Gobara, Hirooka, Shiojiri 399-078I, Nagano, Japan

Tel/fax +8I 263512210

Email ohta@po.mdu.ac.jp
Purpose: The purpose of this study was to demonstrate a displacement of the foveal depression toward the optic disk after idiopathic macular hole (MH) surgery with internal limiting membrane (ILM) peeling.

Methods: Two patients with a unilateral MH developed an MH in the fellow eyes. Vitrectomy with ILM peeling was performed on the fellow eye to close the MH. Images of spectral-domain optical coherence tomography (SD-OCT) were used to measure the disk-to-fovea distances pre MH formation, after MH formation, and 6 months after the closure of the MH.

Results: The disk-to-fovea distance was shorter at 6 months than after the development of the MH $(4,109 \mu \mathrm{m}$ and 4,174 $\mu \mathrm{m}$ in Case 1 and 4,001 $\mu \mathrm{m}$ and 4,051 $\mu \mathrm{m}$ in Case 2).

Conclusion: These results indicate that the fovea moves nasally after the MH surgery with ILM peeling.

Keywords: idiopathic macular hole, internal limiting membrane, optical coherence tomography, pars plana vitrectomy

\section{Introduction}

Kelly and Wendel ${ }^{1}$ reported that idiopathic macular holes (MHs) can be closed by pars plana vitrectomy (PPV) with fluid-gas exchange. Since then, the surgical technique for closing an $\mathrm{MH}$ has been modified, e.g., removal of the internal limiting membrane (ILM), which has increased the anatomic success rates and improved the functional results.

We found earlier that the parafoveal nasal retina was significantly thicker and the temporal retina was significantly thinner than the corresponding areas of the fellow eyes after vitrectomy with removal of the ILM to close an MH. These findings were made from the cross-sectional images of the retina obtained by spectral domain optical coherence tomography (SD-OCT). ${ }^{2}$ In addition, Kumagai et $\mathrm{al}^{3}$ reported that this phenomenon was observed not only in eyes after $\mathrm{MH}$ closure but also after epiretinal membrane (ERM) removal with ILM peeling. These findings indicated that there are alterations in the parafoveal retina after vitrectomy with ILM peeling. The cause for the thickening has not been determined.

Another change in the retinal structure after vitrectomy with ILM peeling is the centripetal movement of the retina around an $\mathrm{MH}$ toward the foveal pit after vitrectomy with ILM peeling. ${ }^{4}$ However, Kawano et $\mathrm{al}^{5}$ recently showed a displacement of the macula toward the optic disc, which has been confirmed in latter studies including ours. $^{6-8}$ These displacements have been measured relative to the location of the $\mathrm{MH}$, 
and how these displacements occur relative to the fovea before the development of an MH has not been determined.

We have had two patients with a unilateral $\mathrm{MH}$ in which the fellow eyes developed an $\mathrm{MH}$. Because both eyes were examined, including SD-OCT recordings, in our standard examination protocol in patients with a unilateral $\mathrm{MH}, \mathrm{SD}-$ OCT images were available for eyes before the $\mathrm{MH}$ formation and also during and after the $\mathrm{MH}$ was closed by vitrectomy with ILM peeling. This was important because the distance between the fovea and optic disc could be measured before, during, and after the closure of an $\mathrm{MH}$ in these two fellow eyes.

\section{Methods}

This was a retrospective study of two patients who had a unilateral $\mathrm{MH}$ and had SD-OCT images recorded from the affected eyes after the $\mathrm{MH}$ formed, from the normal fellow eyes before an $\mathrm{MH}$ formed, from eyes during the $\mathrm{MH}$ formation, and from eyes after the $\mathrm{MH}$ was closed by vitrectomy with ILM peeling. They were both examined at the Matsumoto Dental University Hospital, and all of the procedures adhered to the tenets of the Declaration of Helsinki. The protocol of this study was approved by the institutional review board and ethics committee of Matsumoto Dental University, and a written informed consent for the examination and surgery was obtained from all of the patients. In addition, permission was obtained from both patients to use the findings and any accompanying images in future medical publications with assurance of anonymity.

The best-corrected visual acuity (BCVA) and intraocular pressure (IOP) were measured, and slit-lamp examinations and fundus examinations were performed. Examinations of SD-OCT were performed using the Spectralis HRA+OCT (Heidelberg Engineering, Heidelberg, Germany).

The SD-OCT examinations were performed before, after the $\mathrm{MH}$ formation, and at 6 months after $\mathrm{MH}$ closure by vitrectomy with ILM peeling. The horizontal and vertical images that were centered on the fovea in the cross-hair mode $\left(30^{\circ}\right)$ were evaluated. We determined the site of the foveal pit (Figure 1) or the center of the opened MH manually in the enlarged images of the retina on a monitor screen. We also determined the site of a small retinal vessel crossing the temporal-inferior edge of the optic disk. We then manually measured the distance from the selected site on the disk margin to the selected center of the opened or closed $\mathrm{MH}$ as the disk-to-fovea distance as described in more detail. ${ }^{8}$ Other groups have also reported on the papillofoveal distance by using similar methods. ${ }^{6,7}$

\section{Results}

Two patients underwent vitrectomy with ILM peeling for a unilateral $\mathrm{MH}$, and the demographics of the two patients are shown in Table 1.

\section{Case I}

At the time of the vitrectomy for the left eye, the right eye did not have an $\mathrm{MH}$ (Figure 1). Four months after the surgery on the left eye, a stage $3 \mathrm{MH}$, according to the Gass ${ }^{9}$ classification, developed in the right eye (Figure 2). Her BCVA was 20/32 in the right eye, and the maximum diameter of the $\mathrm{MH}$ was $308 \mu \mathrm{m}$ in the SD-OCT images. Vitrectomy with ILM peeling was performed with standard 25-gage instruments by a single surgeon (KO). Phacoemulsification and implantation of a posterior chamber intraocular lens (IOL) were done. After core vitrectomy, a posterior vitreous detachment (PVD) was created by suction with the vitrectomy cutter after triamcinolone acetonide (TA; MaQaid ${ }^{\circledR}$; Wakamoto Pharmaceuticals Co., Ltd, Tokyo, Japan) was injected into the vitreous cavity. ${ }^{2,8}$ The ILM was made more visible by the TA and grasped at the temporal raphe and

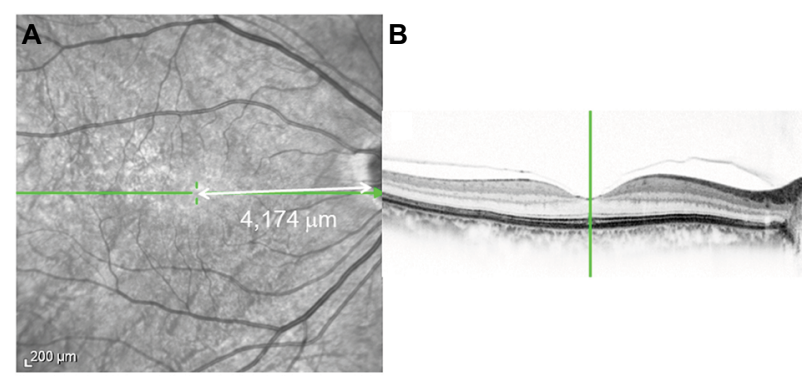

Figure I Infrared image (A) and SD-OCT image (B) of a 66-year-old woman before the opening of an $\mathrm{MH}$.

Notes: The center of the fovea (vertical green line) in a horizontal section (B) was identified in the near-infrared image (green crossbar; $\mathbf{A}$ ). The distance of the diskto-fovea is represented by the white arrow $(\mathbf{A})$.

Abbreviations: SD-OCT, spectral-domain optical coherence tomography; $\mathrm{MH}$, macular hole.

Table I Patient characteristics

\begin{tabular}{lll}
\hline $\begin{array}{l}\text { Demography and clinical characteristics } \\
\text { of eyes }\end{array}$ & Case I & Case 2 \\
\hline Age (years) & 66 & 67 \\
Gender & Female & Male \\
Eye & Right & Left \\
Duration after ipsilateral MH surgery (months) & 4 & 9 \\
Preoperative VA & $20 / 32$ & $20 / 32$ \\
MH stage & 3 & 3 \\
MH size ( $\mu$ m) & 308 & 196 \\
Surgery & $25 G$ PPV & $25 G$ PPV \\
Intraoperative adjuvant & TA & BBG \\
Postoperative VA & $20 / 16$ & $20 / 16$ \\
\hline
\end{tabular}

Abbreviations: BBG, brilliant blue $\mathrm{G}$; $\mathrm{MH}$, macular hole; PPV, pars plana vitrectomy; TA, triamcinolone acetonide; VA, visual acuity. 
peeled $\sim 1.5 \times 2.0$ disk diameters (DDs) around the $\mathrm{MH}$. Then, fluid-air exchange was performed with the air pressure set at $35 \mathrm{mmHg}$. In all, $20 \%$ sulfur hexafluoride $\left(\mathrm{SF}_{6}\right)$ was used for the gas tamponade. The patient was instructed to remain in a prone position for at least 7 days. The $\mathrm{MH}$ was closed after the initial surgery, and the BCVA was 20/16 at 6 months after the surgery.

\section{Case 2}

The preoperative BCVA was 20/32 in the affected eye with a stage $3 \mathrm{MH}$. The size of the $\mathrm{MH}$ was $196 \mu \mathrm{m}$. A similar MH surgery was done by staining the ILM with Brilliant Blue G (Brilliant Peel ${ }^{\circledR}$; Geuder, Heidelberg, Germany). The ILM was peeled $1.5 \times 1.5$ DDs. The BCVA at 6 months was 20/16

The disk-to-fovea distance was $4,174 \mu \mathrm{m}$ pre $\mathrm{MH}$ formation, 4,390 $\mu \mathrm{m}$ after the MH formed, and 4,109 $\mu \mathrm{m}$ at 6 months after the $\mathrm{MH}$ was closed by vitrectomy with ILM peeling in Case 1 (Figure 2). Similarly, the same distances were 4,051 $\mu \mathrm{m}$ before the $\mathrm{MH}$ formed, 4,052 $\mu \mathrm{m}$ after the $\mathrm{MH}$ formed, and 4,001 $\mu \mathrm{m}$ after the $\mathrm{MH}$ was closed by vitrectomy with ILM peeling in Case 2 (Figure 2).

The disk-to-fovea distances were measured by three independent examiners. The mean differences are shown in Table 2. The mean difference of the disk-to-fovea distances at pre $\mathrm{MH}$ formation and at $\mathrm{MH}$ formation was larger than that at $\mathrm{MH}$ closure.

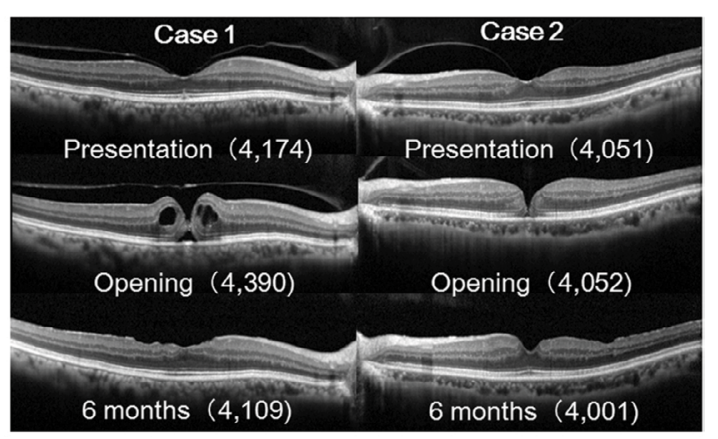

Figure 2 The correlation between changes in SD-OCT and disk-to-fovea distance measurements.

Notes: The distances of disk-to-fovea are presented in parentheses $(\mu \mathrm{m})$. The left figures are the horizontal SD-OCT images of right eye of Case I. The right figures are those of left eye of Case 2.

Abbreviations: SD-OCT, spectral-domain optical coherence tomography; OCT, optical coherence tomography.

Table 2 The mean difference of disc-to-fovea distance, $\mu \mathrm{m}$ (SD)

\begin{tabular}{lll}
\hline & Case I & Case 2 \\
\hline MH formation - pre MH formation & $257.7(63.7)$ & $-3.7(6.5)$ \\
MH formation - MH closure & $327.0(5 \mathrm{I} .7)$ & $68.7(34.1)$ \\
Pre MH formation - MH closure & $69.3(19.9)$ & $72.3(32.0)$ \\
\hline
\end{tabular}

Note: The data are the mean values of three examiners.

Abbreviation: $\mathrm{MH}$, macular hole.
We also merged the infrared images obtained before the $\mathrm{MH}$ formed and after it was closed in both cases (Figure 3). The center of foveal depression was easily determined in the optical coherence tomography (OCT) images (Figure 2). The measurements showed that before the $\mathrm{MH}$ formed (vertical green bar), the fovea moved toward the optic disk after the $\mathrm{MH}$ was closed (vertical white bar) in both cases.

\section{Discussion}

Kawano et $\mathrm{al}^{5}$ reported a displacement of the center of the fovea toward the optic disk after successful closure of an $\mathrm{MH}$ by vitrectomy with ILM peeling. Other groups including ours confirmed this anatomical change after MH surgery. ${ }^{6-8}$ However, this displacement was a shift of the center of an opened $\mathrm{MH}$, and evidence is not available on the site of the preopened $\mathrm{MH}$, the opened $\mathrm{MH}$, and the closed $\mathrm{MH}$ after a closure by vitrectomy with ILM peeling.

We were able to obtain SD-OCT images of the preopened $\mathrm{MH}$ in these two cases because they had an earlier $\mathrm{MH}$ in the fellow eye. Yanagita et $\mathrm{al}^{4}$ studied 21 cases of an $\mathrm{MH}$, and they reported that the retinal blood vessels near the $\mathrm{MH}$ were displaced toward the center of the fovea after a closure of a $\mathrm{MH}$ by vitrectomy with ILM peeling. They suggested that the retina around the $\mathrm{MH}$ moved toward the foveal pit after the MH surgery. Yanagita et al determined the fovea or the fixation point by scanning laser ophthalmoscopic microperimetry.

Kawano et $\mathrm{al}^{5}$ examined the SD-OCT images of 54 eyes with an $\mathrm{MH}$ before and after a closure of the $\mathrm{MH}$. They showed a displacement of the center of the fovea toward the optic disk with an average distance of 0.09 DDs. We also reported that the mean disk-to-fovea distance was significantly shorter by $135.5 \pm 133.8 \mu \mathrm{m}$ than at the baseline at 6 months after the vitrectomy. If the diameter of the disk is set to $1,500 \mu \mathrm{m}$, our results would be comparable to the

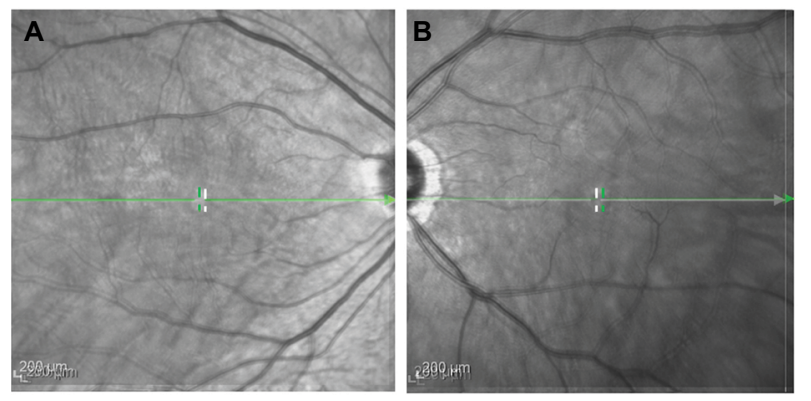

Figure 3 The merged infrared images obtained from preopened and closed $\mathrm{MH}$ of both cases: (A) Case I and (B) Case 2.

Notes: The foveal pits in the preopened $\mathrm{MH}$ are shown as vertical green bars. The center of closed $\mathrm{MH}$ is shown as vertical white bars.

Abbreviation: $\mathrm{MH}$, macular hole. 
values of Kawano et al. Other Japanese groups have also reported similar retinal displacements toward the optic disk after ILM peeling. ${ }^{6,7}$

The papillofoveal distance was also decreased after vitrectomy with ILM peeling in eyes with diabetic macular edema. ${ }^{10}$ However, this macular movement toward the optic disk was not observed in eyes without ILM peeling. These findings would have been expected from the findings in our two cases. The mean distance from the optic disk to the intersection of the nasal vessel in eyes with closed $\mathrm{MH}$ was shorter than that of that in fellow eyes. ${ }^{7}$

It was not determined why the fovea moves toward the optic disk after vitrectomy with ILM peeling. The lower elasticity of retina due to microtubules and actin filaments in the nerve fibers ${ }^{10,11}$ and the contractile properties by actomyosin interaction ${ }^{10,12}$ have been suggested to be the cause. The twisting course of the nerve fiber layer after ILM peeling or asymmetry of the biomechanical forces has also been suggested. ${ }^{10}$ The nerve fibers in the retina would move toward the optic disk after shrinkage of the fibers because the optic nerve fibers are fixed to the lamina cribrosa. ${ }^{13}$

Another possibility is the fact that the MH opens eccentrically. As observed in these two cases, the size of $\mathrm{MH}$ was relatively small, and it is difficult to determine the center of an opened MH. Further large studies on a larger number of patients are needed.

\section{Conclusion}

We found that the postoperative fovea moves nasally relative to the original position after vitrectomy with ILM peeling in two cases. Although this may have been expected from the results of earlier studies, our findings in these two cases demonstrate this phenomenon clearly. Further studies are needed including studies with a larger number of patients.

\section{Acknowledgment}

We thank Prof. Duco Hamasaki of the Bascom Palmer Eye Institute for editing the manuscript.

\section{Disclosure}

The authors report no conflicts of interest in this work.

\section{References}

1. Kelly NE, Wendel RT. Vitreous surgery for idiopathic macular holes: results of a pilot study. Arch Ophthalmol. 1991;109(5):654-659.

2. Ohta K, Sato A, Fukui E. Retinal thickness in eyes with idiopathic macular hole after vitrectomy with internal limiting membrane peeling. Graefes Arch Clin Exp Ophthalmol. 2013;251(5):1273-1279.

3. Kumagai K, Ogino N, Furukawa M, et al. Retinal thickness after vitrectomy and internal limiting membrane peeling for macular hole and epiretinal membrane. Clin Ophthal. 2012;6:679-688.

4. Yanagita T, Shimizu K, Fujimura F, Takano M. Fixation point after successful macular hole surgery with internal limiting membrane peeling. Ophthalmic Surg Lasers Imaging. 2009;40(2):109-114.

5. Kawano K, Ito Y, Kondo M, et al. Displacement of foveal area toward optic disc after macula hole surgery with internal limiting membrane peeling. Eye. 2013;27(7):871-877.

6. Itoh Y, Inoue M, Rii T, Hirota K, Hirakata A. Asymmetrical recovery of cone outer segment tips line and foveal displacement after successful macular hole surgery. Invest Ophthalmol Vis Sci. 2014;55(5):3003-3011.

7. Ishida M, Ichikawa Y, Higashida R, Tsutsumi Y, Ishikawa A, Imamura Y. Retinal displacement toward optic disc after internal limiting membrane peeling for idiopathic macular hole. Am J Ophthalmol. 2014;157(5):971-977.

8. Ohta K, Sato A, Fukui E. Idiopathic macular hole displaced toward optic disc after vitrectomy with internal limiting membrane peeling. J Clin Exp Ophthalmol. 2014;5:332.

9. Gass JD. Reappraisal of biomicroscopic classification of stages of development of a macular hole. Am J Opthalmol. 1995;119(6):752-759.

10. Yoshikawa M, Murakami T, Nishijima K, et al. Macular migration toward the optic disc after inner limiting membrane peeling for diabetic macular edema. Invest Ophthalmol Vis Sci. 2013;54(1):629-635.

11. Conde C, Caseres A. Microtubule assembly, organization and dynamics in axons and dendrites. Nat Rev Neurosci. 2009;10(5):319-332.

12. Gallo G, Yee HF Jr, Letourneau PC. Actin turnover is required to prevent axon retraction driven by endogenous actomyosin contractility. $J$ Cell Biol. 2002;158(7):1219-1228.

13. Hogan MJ, Alvarado JA, Weddell JE. Histology of the Human Eye. Philadelphia: W.B. Saunders Company; 1971:523-606.
International Medical Case Reports Journal

\section{Publish your work in this journal}

The International Medical Case Reports Journal is an international, peer-reviewed open-access journal publishing original case reports from all medical specialties. Previously unpublished medical posters are also accepted relating to any area of clinical or preclinical science. Submissions should not normally exceed 2,000 words or

\section{Dovepress}

4 published pages including figures, diagrams and references. The manuscript management system is completely online and includes a very quick and fair peer-review system, which is all easy to use. Visit $\mathrm{http}: / /$ www.dovepress.com/testimonials.php to read real quotes from published authors. 